\title{
Calcium-Activated Potassium Channels Are Selectively Coupled to P/Q-Type Calcium Channels in Cerebellar Purkinje Neurons
}

\author{
Mary D. Womack, Carolyn Chevez, and Kamran Khodakhah \\ Department of Neuroscience, Albert Einstein College of Medicine, Bronx, New York 10461
}

\begin{abstract}
Cerebellar Purkinje neurons fire spontaneously in the absence of synaptic transmission. P/Q-type voltage-gated calcium channels and calcium-activated potassium channels are required for normal spontaneous activity. Blocking P/Q-type calcium channels paradoxically mimics the effects of blocking calcium-activated potassium channels. Thus, an important function of the $\mathrm{P} / \mathrm{Q}$-type calcium channels is to provide calcium for activation of calcium-activated potassium channels. Purkinje neurons express several classes of voltage-gated calcium channels, and the $\mathrm{P} / \mathrm{Q}$ - and T-type channels make comparable contributions to total calcium entry after an action potential. Here we demonstrate that calcium-activated potassium channels are activated exclusively by calcium entering through P/Q-type voltage-gated calcium channels. This selective coupling is maintained even when calcium flux through voltage-gated channels is increased by increasing the extracellular calcium concentration. Small decreases in $\mathrm{P} / \mathrm{Q}$ current density are likely to alter spontaneous activity of Purkinje neurons via decreased recruitment of calcium-activated potassium channels. In both human and murine animal models, mutations that decrease $\mathrm{P} / \mathrm{Q}$ current density in Purkinje neurons also cause cerebellar ataxia. Alterations in the spontaneous activity of Purkinje neurons may be an important contributing factor to the ataxia in these subjects.
\end{abstract}

Key words: action potential; cerebellum; Purkinje cell; calcium channel; calcium-activated potassium channel; spontaneous firing; AHP; coupling

\section{Introduction}

Signals for motor coordination and balance are coded in the rate and pattern of firing of cerebellar Purkinje neurons, the only neurons that project out of the cerebellar cortex (Ito, 1984). The activity of a Purkinje neuron is controlled both by synaptic input and by intrinsic conductances that cause it to fire spontaneously (Nam and Hockberger, 1997; Raman and Bean, 1999; Womack and Khodakhah, 2002a; Swensen and Bean, 2003). Both P/Q-type calcium channels and calcium-activated potassium channels are important for controlling spontaneous activity in Purkinje neurons (Womack and Khodakhah, 2002a, 2003). A primary function for voltage-gated calcium channels in controlling spontaneous activity appears to be to provide calcium for activation of calcium-activated potassium channels. In dissociated Purkinje neurons, it has been shown that the net calcium-dependent current activated by action potentials in Purkinje neurons is an outward current (Raman and Bean, 1999). Block of P/Q-type calcium channels in spontaneously firing Purkinje neurons results in a significant increase in firing rate and bursting (Womack and

\footnotetext{
Received July 19, 2004; revised Aug. 12, 2004; accepted Sept. 1, 2004.

This work was supported by the New City Speaker's Fund for Biomedical Research and the National Institutes of Health.

Correspondence should be addressed to Kamran Khodakhah, Department of Neuroscience, Albert Einstein College of Medicine, 506 Kennedy Center, 1410 Pelham Parkway South, Bronx, NY 10461. E-mail: Kkhodakh@AECOM.YU.EDU.

D0I:10.1523/JNEUROSCI.2915-04.2004

Copyright $\odot 2004$ Society for Neuroscience $\quad 0270-6474 / 04 / 248818-05 \$ 15.00 / 0$
}

Khodakhah, 2002a), a response similar to that observed after block of calcium-activated potassium channels (Edgerton and Reinhart, 2003; Womack and Khodakhah, 2003).

In many cells, calcium-activated potassium channels are selectively coupled to specific classes of voltage-gated calcium channels (Davies et al., 1996; Marrion and Tavalin, 1998; Prakriya and Lingle, 1999; Smith et al., 2002; Wolfart and Roeper, 2002). Because voltage-gated calcium channels provide the primary source of calcium for activation of calcium-activated potassium channels in Purkinje neurons, the kinetics and voltage dependence of activation will be limited by the kinetics and voltage dependence of the coupled voltage-gated calcium channels. Purkinje neurons express functional P/Q-, T-, R-, N-, and L-type calcium channels. P/Q-type channels comprise $\sim 80 \%$ of the high-threshold current, the remainder being equally divided between N- and L-type (Regan, 1991; Mintz et al., 1992). Lowthreshold channels ( $\mathrm{T}$ - or R-type calcium channels) are also present (Regan, 1991; McDonough and Bean, 1998; Pouille et al., 2000). The main voltage-gated calcium channels activated by an action potential waveform, however, are T- and P/Q-type (Raman and Bean, 1999; Swensen and Bean, 2003). P/Q-type channels contribute a large current that rapidly deactivates after action potential repolarization. Although the peak calcium current provided by T-type channels is much less, T-type channels deactivate more slowly so that significant T-type current is active during the interspike intervals (Raman and Bean, 1999; Swensen and Bean, 2003). Integrating the P/Q- and T-type calcium currents reveals 
that, per action potential, as much as $50 \%$ of total calcium enters through T-type channels [cf. Raman and Bean (1999), their Fig. 8b].

Because both calcium-activated potassium channels and voltagegated calcium channels are critical for normal spontaneous firing of Purkinje neurons, we determined which voltage-gated calcium channels are responsible for activating calcium-activated potassium channels. We show that calcium for activating both large $(\mathrm{BK})$ and small (SK) conductance calcium-activated potassium channels is provided solely by $\mathrm{P} / \mathrm{Q}$-type calcium channels.

\section{Materials and Methods}

Preparation of slices. Wistar rats at 15-30 d of age were anesthetized with halothane and killed by decapitation. Sagittal slices (300- $\mu$ m-thick) were prepared from the cerebellum using a vibratome (Campden Instruments, Loughborough, UK). Slices were maintained at room temperature in the recording solution until use (1-8 hr).

Recording and analysis. Slices were mounted in a chamber on the stage of an upright Zeiss (Oberkochen, Germany) microscope and visualized using a $40 \times$ water immersion objective with infrared optics. Slices were continuously superfused at a rate of $1.5 \mathrm{ml} / \mathrm{min}$ with recording solution containing (in mM): $125 \mathrm{NaCl}, 2.5 \mathrm{KCl}, 26 \mathrm{NaHCO}_{3}, 1.25 \mathrm{NaH}_{2} \mathrm{PO}_{4}, 1$ $\mathrm{MgCl}_{2}, 2 \mathrm{CaCl}_{2}$, and 10 glucose, $\mathrm{pH} 7.4$ when gassed with $5 \% \mathrm{CO}_{2}-95 \%$ $\mathrm{O}_{2}$. To block rapid synaptic transmission, the recording solution also contained kynurenic acid ( $5 \mathrm{~mm}$ ), a broad-spectrum ionotropic glutamate receptor antagonist (Stone, 1993), and the $\mathrm{GABA}_{\mathrm{A}}$ antagonist picrotoxin (Yoon et al., 1993). The slice temperature was maintained at $35^{\circ} \mathrm{C}$ $\left( \pm 0.5^{\circ} \mathrm{C}\right)$ by adjusting the temperature of the bathing solution. The volume of the chamber was $2 \mathrm{ml}$, requiring several minutes for complete wash in of the antagonists or blockers. Extracellular recordings were made from individual Purkinje neurons using a home-made differential amplifier with glass pipette electrodes (tip size, $0.3-1 \mu \mathrm{m}$ ) filled with the recording solution. The pipette tip was positioned just above, or lightly touching, the cell body near the axon hillock in which the largest potential changes were usually recorded. Action potentials appeared as fast negative deflections of 50-1000 $\mu \mathrm{V}$. Whole-cell recordings were made with borosilicate glass pipettes (3-6 $\mathrm{M} \Omega$ ) filled with (in $\mathrm{mm}$ ): 140 $\mathrm{K}$-methyl sulfate, $10 \mathrm{KCl}, 5 \mathrm{NaCl}, 2 \mathrm{MgATP}, 0.01 \mathrm{EGTA}$, and 10 HEPES, $\mathrm{pH}$ 7.2. Whole-cell data were recorded using an Optopatch amplifier (Cairn Research Ltd, Faversham, Kent, UK). Data were sampled at 10 $\mathrm{kHz}$ for extracellular recordings and $20 \mathrm{kHz}$ for whole-cell recordings using a National Instruments (MIO-16XE-10; Austin, TX) digital-toanalog-analog-to-digital card and an IBM-compatible computer. Data acquisition and analyses were done with software written in-house using LabView (National Instruments). Kynurenic acid, picrotoxin, and apamin were obtained from Sigma Chemical (St. Louis, MO). Iberiotoxin and $\omega$ conotoxin GVIA were from Tocris Cookson (Ellisville, MO), and $\omega$ conotoxin MVIIC was from Alomone Labs (Jerusalem, Israel). The $\mathrm{EC}_{50}$ for the lot of $\omega$ conotoxin MVIIC used was determined by the supplier to be $200 \mathrm{~nm}$. Mibefradil was a generous gift from Hoffmann-La Roche (Basel, Switzerland). All other chemicals were obtained from Sigma or Fisher Scientific (Fairlawn, NJ). The kinetics of block of calcium channels with toxins can be very slow (McDonough et al., 1996). Therefore, calcium channel antagonists were applied for at least $30 \mathrm{~min}$.

\section{Results \\ Calcium-activated potassium channels that contribute to action potential waveform are coupled to $\mathrm{P} / \mathrm{Q}$-type calcium channels}

The most direct way to examine selective coupling between voltage-gated calcium and calcium-activated potassium channels is to use whole-cell voltage clamp. Voltage clamp of calcium currents in intact Purkinje neurons, however, is impossible because of the presence of a high density of voltage-gated calcium channels in the dendritic tree and the impracticality of space clamping the dendrites. Although voltage clamp of voltage-gated currents is possible in dissociated Purkinje neuron soma, it has been reported that dissociated Purkinje neurons show a large variability a

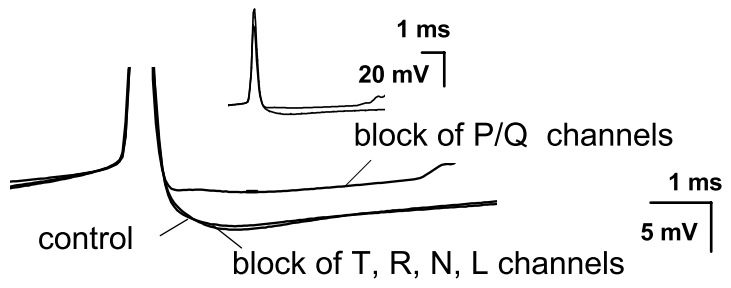

b

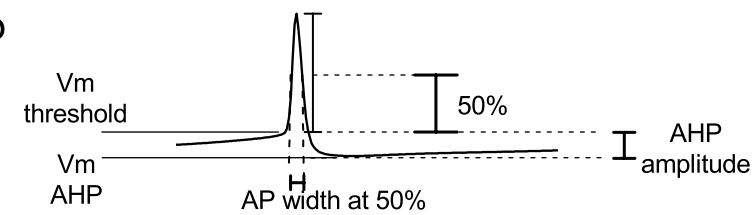

C block of $T, R, N, L$ channels
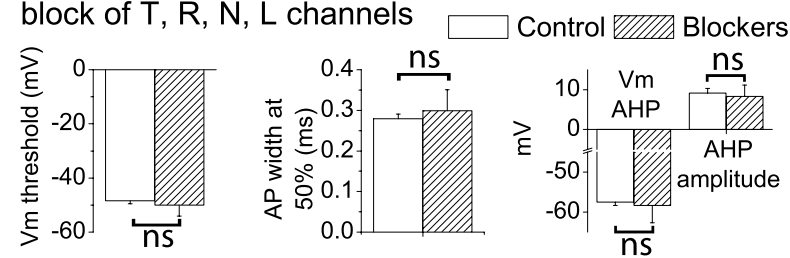

block of $P / Q$ channels
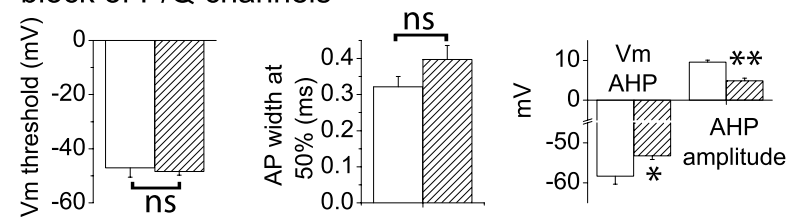

Figure 1. Contribution of voltage-gated calcium channels on the action potential waveform in Purkinje neurons. $a$, Whole-cell current-clamp recordings from a spontaneously firing Purkinje neuron under control conditions in the presence of blockers for $\mathrm{T}_{-}, \mathrm{R}-, \mathrm{N}-$-, and L-type calcium channels or for $\mathrm{P} / \mathrm{Q}$-type calcium channels. The action potential has been truncated to show the AHP more clearly; complete action potentials are shown in the inset. Each trace is the average of 50-100 action potentials. Blockers were mibefradil (1 $\mu \mathrm{m})$ for T- and R-type channels, $\omega$ conotoxin GVIA (3 $\mu \mathrm{m})$ for N-type channels, nimodipine $(2 \mu \mathrm{m})$ for L-type channels, and $\omega$ conotoxin MVIIC $(2 \mu \mathrm{M})$, $\omega$ agatoxin IVA $(1-2 \mu \mathrm{M}), \operatorname{orCd}^{2+}(100 \mu \mathrm{M})$ for P/Q-type channels. $b$, Diagram showing the parameters of the action potential (AP) waveform that were measured. $V_{m}$ threshold, Membrane potential 500 msec before the peak of the action potential; $V_{m} A H P$, membrane potentialat the peak of the AHP; AHP amplitude, $V_{m} A H P-V_{m}$ threshold; AP width at $50 \%$, width of the action potential at one-half the difference between the peak potential and $V_{m}$ threshold. c, Summary of the effects of calcium channel blockers on the action potential waveform. Values were significantly different for ${ }^{*} p<0.05$ and ${ }^{* *} p<0.005$ by one-way ANOVA. Differences between all other values were not significant (ns). Error bars are \pm SEM; $n=7$.

in the expression of SK currents (Khaliq et al., 2003). This finding is in contrast to intact Purkinje neurons in slices in which block of SK channels caused a large increase in firing rate and a decrease in the afterhyperpolarization (AHP) in every neuron tested (Womack and Khodakhah, 2002a; Edgerton and Reinhart, 2003).

We therefore measured action potential AHPs to investigate the selective coupling of calcium-activated potassium channels and voltage-gated calcium channels. In Purkinje neurons, activation of both SK and BK channels is time locked to the action potential. Both channels contribute an AHP that follows each action potential but makes little contribution to action potential repolarization or to resting membrane potential (Womack and Khodakhah, 2002b, 2003; Edgerton and Reinhart, 2003; Sausbier et al., 2004). It is possible, therefore, to use AHPs measured with whole-cell current-clamp recording to determine which classes of voltage-gated calcium channels provide calcium for activation of calcium-activated potassium channels. Action potential waveform parameters (Fig. 1b) were compared in the presence and absence of blockers of specific classes of voltage-gated calcium 
channels. Block of P/Q-type channels caused a decrease in the AHP amplitude (Fig. 1a-c) with no effect on action potential threshold or width (Fig. 1a,c), an effect similar to that seen after block of calcium-activated potassium channels. In contrast, simultaneous block of T-, N-, R-, and L-type voltage-gated calcium channels had no effect on action potential waveform (Fig. 1a-c). These results confirm and extend those of Edgerton and Reinhart (2003) and establish that SK and BK channels that contribute to the AHP are selectively coupled to P/Q-type voltagegated calcium channels.

Calcium-activated potassium channels that contribute to spontaneous firing are selectively coupled to P/Q-type calcium channels

Coupling between voltage-gated calcium channels and calcium-activated potassium channels is heavily influenced by intracellular calcium buffers that may be washed out in the whole-cell recording configuration (Roberts, 1994; Naraghi, 1997). To investigate the functional coupling of voltage-gated calcium channels to calcium-activated potassium channels without altering cytoplasmic constituents, we tested the effects of specific calcium channel blockers on spontaneous activity using single-cell extracellular recording. The effects of the calcium channel blockers were compared with those of blockers of calcium-activated potassium channels. We compared the rate and regularity of spontaneous firing. As a measure of regularity of firing, we calculated the coefficient of variation of interspike intervals collected during a $5 \mathrm{sec}$ recording period. Block of T- and R-type (Fig. 2a) or $\mathrm{N}-$ and L-type (Fig. 2b) voltage-gated calcium channels had no effect on the rate or regularity of spontaneous firing. In contrast, blocking only onehalf of the P/Q-type voltage-gated calcium channels (corresponding to removal of $\sim 25 \%$ of total action potential-evoked calcium entry) caused a significant increase in the rate and decrease in the regularity of spontaneous firing (Fig. 2c), effects similar to those observed after block of SK channels. The slow time course of block of P/Q channels with $\omega$ contotoxin MVIIC, particularly at the low concentrations used here, is well documented (McDonough et al., 1996). Higher concentrations result in more rapid block and similarly affect the firing rate of Purkinje cells within a few minutes (Womack and Khodakhah, 2002a). These results show that under physiological conditions, calcium-activated potassium channels are selectively coupled to P/Q-type calcium channels.

Selective coupling between $K_{\mathrm{Ca}}$ and P/Q-type channels remains under conditions of increased calcium influx Calcium flux through a channel causes a local increase in intracellular calcium that falls off within a short distance from the channel (Fogelson and Zucker, 1985; Roberts, 1993; Naraghi,

\section{a block of T-type channels}

b block of N- and L-type channels
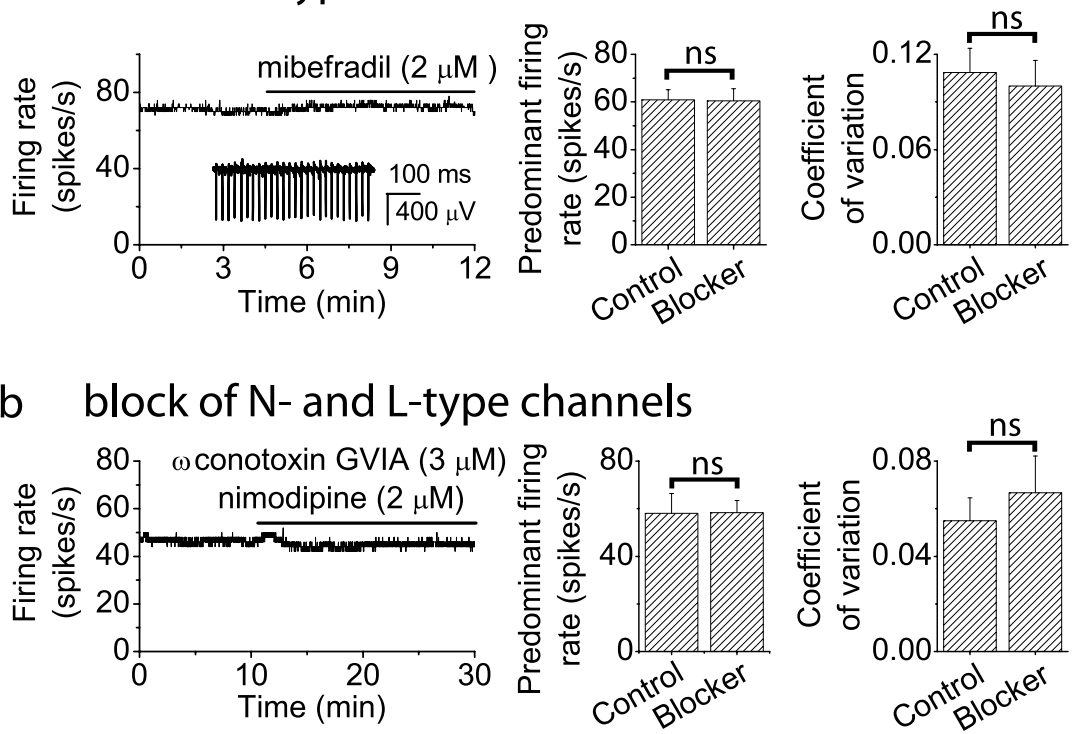

c block of P/Q-type channels
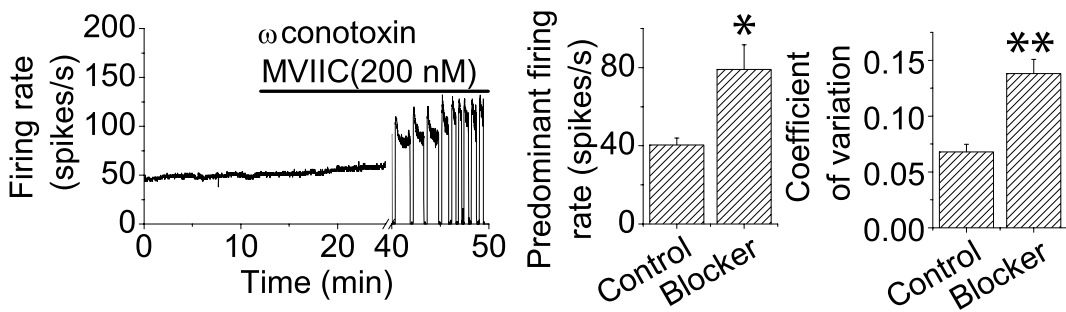

Figure 2. Effect of blockers of voltage-gated calcium channels on spontaneous firing in Purkinje neurons. Spontaneous firing of individual Purkinje neurons was monitored with extracellular recording. $a$, Block of T- and R-type calcium channels does not Histograms comparing average firing rate and coefficient of variation of interspike intervals under control conditions and after rate. Left, Average firing rate versus time for a single neuron. Right, Histograms comparing average firing rate and coefficient of

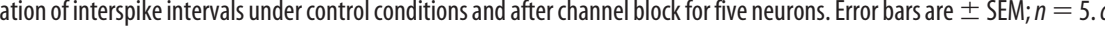
Average firing rate versus time for a single neuron. Block of $\mathrm{P} / 0$ channels increased the firing rate and caused the cell 列 comparing average firing rate and coefficient of variation of interspike intervals under control conditions and after channel block. Error bars are $\pm \mathrm{SEM} ; n=5$. Values were significantly different for ${ }^{*} p<0.02$ and ${ }^{* *} p<0.005$ by one-way ANOVA. Other differences were not significant (ns).

1997). Selective coupling can be achieved by localization of calcium-activated potassium channels near calcium channels within the region of calcium increase. The region of effective calcium increase can be enlarged by increasing calcium influx because calcium concentration at a fixed distance from the channel is proportional to the calcium flux through the channel (Roberts, 1993). Therefore, by increasing the calcium influx, it is possible to recruit calcium-activated potassium channels at a farther distance from the channel. We asked whether calcium-activated potassium channels remain selectively coupled to P/Q-type calcium channels under conditions in which calcium influx through individual calcium channels is increased. Experiments were first performed in the presence of apamin (100 nM), a specific blocker of SK channels. Increasing the extracellular calcium concentration from 2 to $5 \mathrm{~mm}$, which has been estimated to increase calcium influx through voltage-gated channels by $\sim 40 \%$ (Almers 
a

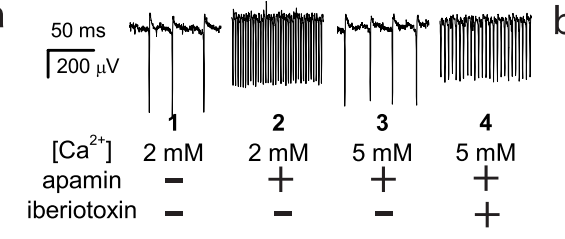

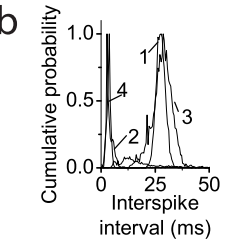

ns
C
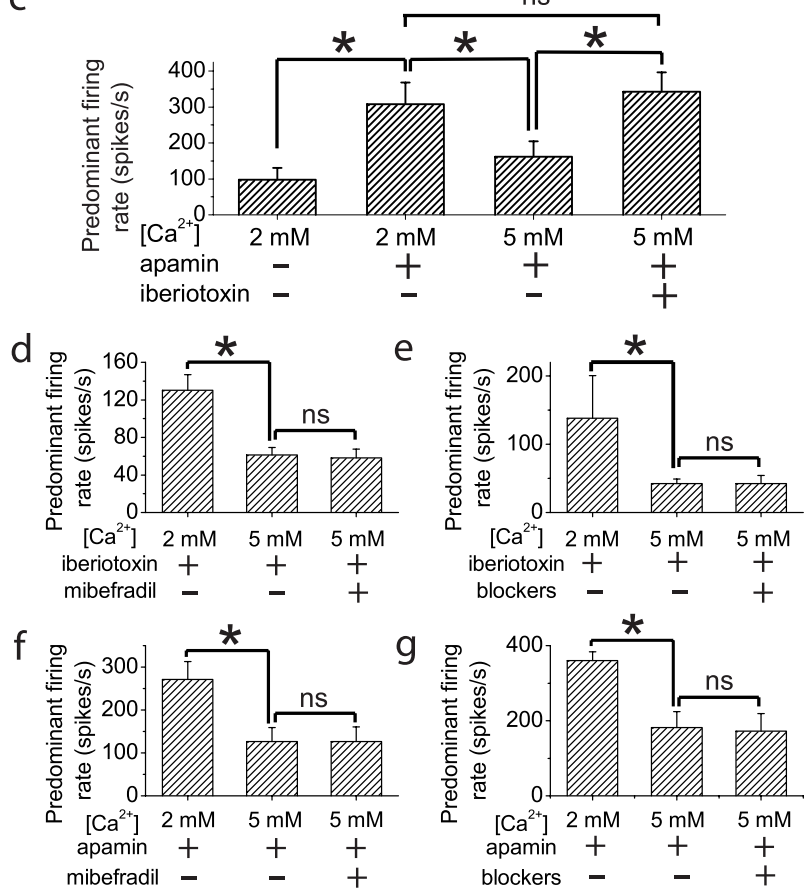

Figure 3. Effect of increased calcium entry on selective coupling between calcium channels and calcium-activated potassium channels. $a-c$, Increasing the extracellular calcium concentration decreases the firing rate by activating BK channels. $a$, Spontaneous activity recorded from a Purkinje neuron. After SK channels were blocked with apamin (100 nM), increasing the extracellular calcium concentration from 2 to $5 \mathrm{~mm}$ decreased the firing rate. The firing rate decrease was reversed by blocking BK channels with iberiotoxin (100 nm). b, Distribution of interspike intervals for the cell in $a . c$, Average of the predominant firing rate from five Purkinje neurons. Error bars are $\pm S E M ; n=5$. d,e, Activation of SK channels by increased extracellular calcium concentration was not reversed by block of T- and R-type $(d)$ or combined block of T-, $\mathrm{R}-, \mathrm{N}$-, and L-type calcium channels (e). All recordings were made in the presence of iberiotoxin to block BK channels. Error bars are \pm SEM; $n=7(d)$ and $n=6(e) . f, g$, Activation of BK channels by increasing extracellular calcium concentration was not reversed by block of $\mathrm{T}$ - and R-type ( $f$ ) or combined block of T-, R-, N-, and L-type calcium channels $(g)$. All recordings were made in the presence of apamin to block SK channels. Error bars are \pm SEM; $n=6(f)$ and $n=$ $6(g)$. Values were significantly different for ${ }^{*} p<0.05$ by one-way ANOVA. Other differences were not significant (ns).

and McCleskey, 1984), decreased the average firing rate to approximately one-half the value observed in apamin (Fig. $3 a-c$ ). The decrease was not caused by surface charge screening because it was not mimicked by increasing the extracellular magnesium concentration from 1 to $4 \mathrm{~mm}$ (data not shown). Indeed, the decrease in firing rate resulted from recruitment of BK channels because it was reversed by application of iberiotoxin (100 nM), a specific BK channel blocker (Fig. 3a-c). In similar experiments, performed in the continuous presence of $100 \mathrm{~nm}$ iberiotoxin, increasing the extracellular calcium concentration decreased the firing rate by $\sim 50 \%$, and the decrease was reversed by application of $100 \mathrm{~nm}$ apamin (data not shown). Together, these experiments demonstrate that increasing the extracellular calcium concentration decreases the firing rate by recruiting additional BK and SK channels and allowed us to ask whether, with increased calcium influx, BK and SK channels remain selectively coupled to P/Qtype calcium channels. Selective coupling of BK and SK channels were addressed in separate experiments. First, experiments were performed in the continuous presence of iberiotoxin to block BK channels. Increasing the extracellular calcium decreased the firing rate (Fig. $3 d, e$ ) as a result of additional recruitment of SK channels. To determine whether the recruitment was attributable to increased calcium flux through non-P/Q-type calcium channels, mibefradil, a specific blocker of T-type calcium channels (Fig. 3d), or a mixture of blockers for T-, R-, N-, and L-type channels (Fig. 3e) were applied. None of these blockers reversed the high calciuminduced decrease in firing rate (Fig. $3 d, e$ ). In the presence of apamin, a blocker of SK channels, increasing the extracellular calcium concentration decreased the firing rate by recruiting additional $\mathrm{BK}$ channels. Application of mibefradil (Fig. $3 f$ ) or combined blockers for T-, R-, N-, and L-type channels (Fig. 3g) failed to reverse the firing rate decrease. Thus, even under conditions of increased calcium influx, both BK and SK channels remain selectively coupled to P/Q-type calcium channels.

\section{Discussion}

Here we show that activation of calcium-activated potassium channels, which control spontaneous firing in Purkinje neurons, is mediated through $\mathrm{P} / \mathrm{Q}$-type calcium channels. The selective coupling remains even when calcium flux through individual calcium channels is increased by increasing the extracellular calcium concentration. Selective coupling of calcium-activated potassium channels to specific classes of voltage-gated calcium channels can be achieved by localization of the calcium-activated potassium channels near the calcium channels within the region in which the calcium increases locally when the calcium channel opens. Although in Purkinje neurons such a mechanism may explain the coupling of $\mathrm{P} / \mathrm{Q}$-type calcium channels with BK channels that have a relatively low affinity for calcium and are unlikely to be significantly activated at resting calcium levels, it does not provide a plausible explanation for selective coupling of SK channels to P/Q-type calcium channels. This is because activation of voltage-gated calcium channels during spontaneous firing increases the average cytoplasmic calcium concentration. It has been estimated that in Purkinje neurons, a train of action potentials increases the average cytoplasmic calcium concentration by 50-100 nм from resting levels of 10-50 nм (Lev-Ram et al., 1992), with the increase immediately beneath the plasma membrane expected to be even larger (Eilers et al., 1995). As much as one-half of this increase is likely to be attributable to T-type calcium channels because a significant fraction of the total calcium entry with each action potential is provided by T-type calcium channels [cf. Raman and Bean (1999), their Fig 8b; Swensen and Bean (2003)]. Although calcium changes over this range are unlikely to activate BK channels, which even at extremely depolarized membrane potentials have a calcium affinity of $\sim 1 \mu \mathrm{M}$ (Womack and Khodakhah, 2002b), they should result in significant activation of SK channels. This is because SK2 channels, the SK channel subtype expressed in Purkinje neurons (Cingolani et al., 2002), have a calcium affinity of $\sim 300 \mathrm{~nm}$ (Bond et al., 1999). Average cytoplasmic calcium increases as a result of activation of T-type calcium channels during spontaneous firing would be expected to recruit SK channels. The fact that this is not observed suggests either that SK channels in Purkinje neurons have a lower affinity than predicted by observations of cloned SK2 channels or that the channels are sequestered with P/Q-type calcium channels in a cellular compartment that is insensitive to the average submembrane calcium concentration in the soma. During firing of sodium- 
dependent action potentials, intracellular calcium increases occur in the proximal dendrites, in the soma, and in the proximal axon (LevRam et al., 1992; Callewaert et al., 1996). One intriguing possibility could be that functional SK channels are primarily localized with $\mathrm{P} / \mathrm{Q}$-type calcium channels in the proximal axon. Isolation of SK channels from the soma may explain the finding that block of SK channels fails to alter the input resistance in spontaneously firing Purkinje neurons (Edgerton and Reinhart, 2003; Womack and Khodakhah, 2003). It may also account for the large variability in SK current density reported for dissociated Purkinje neurons (Khaliq et al., 2003), because there is likely to be cell-to-cell variation in the amount of proximal axon dissociated with the soma. Localization of SK channels in the proximal axon would place the channels where they are optimally located to regulate firing and may serve to uncouple calcium-dependent regulation of spontaneous firing from calcium-dependent regulation of other processes such as long-term changes in excitability or gene expression.

Given the central role of Purkinje neurons in the cerebellar cortex, it is expected that perturbations that alter their spontaneous activity will cause deficits in cerebellar function. It has been recently shown that knock-out or knock-down of either BK (Sausbier et al., 2004) or SK (Shakkottai et al., 2004) channels causes cerebellar ataxia. Ataxia also results from knock-out of an intracellular calcium buffer, present at a high concentration in Purkinje neurons (Airaksinen et al., 1997), which may affect coupling between calcium channels and calcium-activated potassium channels. It is likely that spontaneous firing of Purkinje neurons is greatly altered in these animals. The results presented here demonstrate a role for P/Q-type calcium channels in controlling spontaneous firing of Purkinje neurons via activation of calcium-activated potassium channels. In several human and animal models, mutations that decrease P/Q current density cause cerebellar ataxia (Pietrobon, 2002). The ataxia has typically been attributed to decreased transmitter release resulting from decreased calcium current density at synaptic terminals. However, our results suggest that P/Q channel mutations may also lead to ataxia by perturbing the rate or pattern of spontaneous firing in Purkinje neurons.

\section{References}

Airaksinen MS, Eilers J, Garaschuk O, Thoenen H, Konnerth A, Meyer M (1997) Ataxia and altered dendritic calcium signaling in mice carrying a targeted null mutation of the calbindin D28k gene. Proc Natl Acad Sci USA 94:1488-1493.

Almers W, McCleskey EW (1984) Non-selective conductance in calcium channels of frog muscle: calcium selectivity in a single-file pore. J Physiol (Lond) 353:585-608.

Bond CT, Maylie J, Adelman JP (1999) Small-conductance calciumactivated potassium channels. Ann NY Acad Sci 868:370-378.

Callewaert G, Eilers J, Konnerth A (1996) Axonal calcium entry during fast "sodium" action potentials in rat cerebellar Purkinje neurones. J Physiol (Lond) 495:641-647.

Cingolani LA, Gymnopoulos M, Boccaccio A, Stocker M, Pedarzani P (2002) Developmental regulation of small-conductance $\mathrm{Ca}^{2+}$-activated $\mathrm{K}^{+}$ channel expression and function in rat Purkinje neurons. J Neurosci 22:4456-4467.

Davies PJ, Ireland DR, McLachlan EM (1996) Sources of Ca2 + for different $\mathrm{Ca}(2+)$-activated $\mathrm{K}+$ conductances in neurones of the rat superior cervical ganglion. J Physiol (Lond) 495:353-366.

Edgerton JR, Reinhart PH (2003) Distinct contributions of small and large conductance $\mathrm{Ca} 2+$-activated $\mathrm{K}+$ channels to rat Purkinje neuron function. J Physiol (Lond) 548:53-69.

Eilers J, Callewaert G, Armstrong C, Konnerth A (1995) Calcium signaling in a narrow somatic submembrane shell during synaptic activity in cerebellar Purkinje neurons. Proc Natl Acad Sci USA 92:10272-10276.

Fogelson AL, Zucker RS (1985) Presynaptic calcium diffusion from various arrays of single channels. Implications for transmitter release and synaptic facilitation. Biophys J 48:1003-1017.

Ito M (1984) The cerebellum and neural control. New York: Raven.

Khaliq ZM, Gouwens NW, Raman IM (2003) The contribution of resurgent sodium current to high-frequency firing in Purkinje neurons: an experimental and modeling study. J Neurosci 23:4899-4912.

Lev-Ram V, Miyakawa H, Lasser-Ross N, Ross WN (1992) Calcium transients in cerebellar Purkinje neurons evoked by intracellular stimulation. J Neurophysiol 68:1167-1177.

Marrion NV, Tavalin SJ (1998) Selective activation of Ca2+-activated K+ channels by co-localized Ca2 + channels in hippocampal neurons. Nature 395:900-905.

McDonough SI, Bean BP (1998) Mibefradil inhibition of T-type calcium channels in cerebellar Purkinje neurons. Mol Pharmacol 54:1080-1087.

McDonough SI, Swartz KJ, Mintz IM, Boland LM, Bean BP (1996) Inhibition of calcium channels in rat central and peripheral neurons by omegaconotoxin MVIIC. J Neurosci 16:2612-2623.

Mintz IM, Adams ME, Bean BP (1992) P-type calcium channels in rat central and peripheral neurons. Neuron 9:85-95.

Nam SC, Hockberger PE (1997) Analysis of spontaneous electrical activity in cerebellar Purkinje cells acutely isolated from postnatal rats. J Neurobiol 33:18-32.

Naraghi M (1997) T-jump study of calcium binding kinetics of calcium chelators. Cell Calcium 22:255-268.

Pietrobon D (2002) Calcium channels and channelopathies of the central nervous system. Mol Neurobiol 25:31-50.

Pouille F, Cavelier P, Desplantez T, Beekenkamp H, Craig PJ, Beattie RE, Volsen SG, Bossu JL (2000) Dendro-somatic distribution of calciummediated electrogenesis in Purkinje cells from rat cerebellar slice cultures. J Physiol (Lond) 527:265-282.

Prakriya M, Lingle CJ (1999) BK channel activation by brief depolarizations requires Ca2 + influx throu. J Neurophysiol 81:2267-2278.

Raman IM, Bean BP (1999) Ionic currents underlying spontaneous action potentials in isolated cerebellar Purkinje neurons. J Neurosci 19:1663-1674.

Regan LJ (1991) Voltage-dependent calcium currents in Purkinje cells from rat cerebellar vermis. J Neurosci 11:2259-2269.

Roberts WM (1993) Spatial calcium buffering in saccular hair cells. Nature 363:74-76.

Roberts WM (1994) Localization of calcium signals by a mobile calcium buffer in frog saccular hair cells. J Neurosci 14:3246-3262.

Sausbier M, Hu H, Arntz C, Feil S, Kamm S, Adelsberger H, Sausbier U, Sailer CA, Feil R, Hofmann F, Korth M, Shipston MJ, Knaus HG, Wolfer DP, Pedroarena CM, Storm JF, Ruth P (2004) Cerebellar ataxia and Purkinje cell dysfunction caused by $\mathrm{Ca} 2+$-activated $\mathrm{K}+$ channel deficiency. Proc Natl Acad Sci USA 101:9474-9478.

Shakkottai VG, Chou CH, Oddo S, Sailer CA, Knaus HG, Gutman GA, Barish ME, LaFerla FM, Chandy KG (2004) Enhanced neuronal excitability in the absence of neurodegeneration induces cerebellar ataxia. J Clin Invest 113:582-590.

Smith MR, Nelson AB, Du LS (2002) Regulation of firing response gain by calcium-dependent mechanisms in vestibular nucleus neurons. J Neurophysiol 87:2031-2042.

Stone TW (1993) Neuropharmacology of quinolinic and kynurenic acids. Pharmacol Rev 45:309-379.

Swensen AM, Bean BP (2003) Ionic mechanisms of burst firing in dissociated Purkinje neurons. J Neurosci 23:9650-9663.

Wolfart J, Roeper J (2002) Selective coupling of T-type calcium channels to SK potassium channels prevents intrinsic bursting in dopaminergic midbrain neurons. J Neurosci 22:3404-3413.

Womack M, Khodakhah K (2002a) Active contribution of dendrites to the tonic and trimodal patterns of activity in cerebellar Purkinje neurons. J Neurosci 22:10603-10612.

Womack MD, Khodakhah K (2002b) Characterization of large conductance $\mathrm{Ca} 2+$-activated $\mathrm{K}+$ channels in cerebellar Purkinje neurons. Eur J Neurosci 16:1214-1222.

Womack MD, Khodakhah K (2003) Somatic and dendritic smallconductance calcium-activated potassium channels regulate the output of cerebellar Purkinje neurons. J Neurosci 23:2600-2607.

Yoon KW, Covey DF, Rothman SM (1993) Multiple mechanisms of picrotoxin block of GABA-induced currents in rat hippocampal neurons. J Physiol (Lond) 464:423-439. 